\title{
Improvement Skull Stripping Algorithm of MRI Brain Images Based on Fuzzy Morphological Operation
}

\author{
Shaima Abd El-Kader', Mohamed Morse², Mohy Eldin A. Abo-Elsoud ${ }^{3}$, Rasheed Mokhtar ${ }^{4}$ \\ ${ }^{1}$ Nile Academy for Engineering, Communication department, Egypt, Mansoura \\ ${ }^{2,3,4}$ Mansoura University, Communication Department, Egypt, Mansoura
}

\begin{abstract}
It based on nonsensical filter and fuzzy morphological operation which allow maintaining an edge preserving.Its accuracy has been verified against three state-of-the-art skull stripping method: BSE(Brain surface extraction),BET(brain extraction tool), Robust Brain Extraction (ROBEX).The proposed method tested on two data set to compare result with another paper. The performance of proposed method has been assessed using the jccard scores, sensitivity and specificity as statistical method.The main advantage of proposed method is robust and speed.
\end{abstract}

Keywords: Magnetic Resonance, Skull stripping, nonsensical filter, Fuzzy morphological.

\section{Introduction}

In modern medical imaging applications, Magnetic Resonance (MR) images have become a common approach for providing reliable anatomical information of the human brain. Skull stripping being a preparatory step, intended to dispense with non-cerebrum tissues from MR images for some clinical applications and examinations, its speed and precision are considered as the key elements in segmentation of brain image. Skull stripping is an essential pre-processing venture in numerous medicinal picture applications it intends to evacuate non-cerebrum tissues (e.g., skull, Dura, and scalp) and hold mind parenchyma in MR image to at first separate the complete brain matter from the encompassing non brain portion like dura and skull. Generally misclassifications would happen. For a human viewer it is anything but difficult to utilize the dark cerebrospinal fluid border to distinguish between brain matter and skull. In computer vision this is more muddled, particularly in districts, where this fringe gets thin or even cleans up. Human can undoubtedly handle these scenes. Numerous skullstripping calculations were created. Automatic and manual segmentation are the procedure of dividing the picture into particular locales. The segmentation of the entire cerebrum or the skull-stripping process relies on upon a specific imaging contrast furthermore, on the picture power inhomogeneity. Each of the existing skull-stripping techniques has its shortcomings and embraced in the clinical environment. Today, fully or partially automatic segmentation routines are accepted acknowledged, but heir results firmly rely on upon the hypothetical and calculation models.

Generally speaking, research studied in skull stripping can be broadly divided into three major categories. The first category makes use of a series of morphological operators.

Sajjad Mohsin et.al [1] employed mathematical morphological algorithms for the removal of skull, after the detection of false background. This method fails on low contrast and noisy images analyzing intracranial volumes are the extraction of the brain cortex from T1-weighted MRI head scans. The consequent investigation, tissue division is enormously dependent on the strength and exactness of the brain masks produced in the cerebrum extraction stage. The second class depends on arrangement and grouping calculations. The support vector machine (SVM) is one case of the delegate measurable classifiers adjacent to the regular apparatuses, for example, Bayesian, neural systems, and closest neighbor strategies. The SVM model marks voxels as determining so as to have a place with a remarkable class an arrangement of hyperplanes that amplifies the partition between classes. These techniques maintain a strategic distance from the handling of back ground and skull zones. Be that as it may, not able to handle loud information, haphazardly picking the bunch focus it can't prompt the productive result. Thresholding is one of the most established methods in image division procedure. A binary image can be gotten while thresholding a gray scale image. Rosniza Roslan [2] demonstrated that thresholding the cerebrum could be utilized for skull stripping. Region growing [3] is a locale based image division strategy, likewise called a pixel based image division system on the grounds that it includes the choice of starting seed focuses. This methodology is to segmenting neighboring pixels of initial seed points and determines whether the pixel neighbors should be added to the region. The disadvantage of this algorithm is that user has to select the seed regions and threshold values. F.Segonne et.al [4] proposes a half and half approach that uses for skull stripping. Morphology-Based Methods Generally, these techniques utilize the morphological disintegration and expansion operations to partitioned the skull from the mind area. These systems require a blend of thresholding and edge recognition techniques. The principle downsides of these techniques are that they frequently rely on upon numerous parameters, for example, size and state of the basic component for morphological operation. These parameters are settled by exact experimentation; the worth on these parameters straightforwardly impacts the last yield of these strategies. The technique, programmed recognition of mind 


\section{International Journal of Science and Research (IJSR) \\ ISSN (Online): 2319-7064}

Index Copernicus Value (2013): 6.14 | Impact Factor (2014): 5.611

shapes in MRI datasets created by Brummer et al. [5] is one of the first generally utilized routines for skull stripping. It comprises of histogram-based thresholding and morphological operations. In light of the cerebrum anatomical information, it segregates between the sought and undesired structures. This technique is actualized utilizing a grouping of ordinary and novel morphological operations, utilizing $2 \mathrm{D}$ and $3 \mathrm{D}$ operations. In any case, existing routines that utilization scientific morphology are now and again delicate to little information varieties and it is hard to locate the ideal morphology size for isolating the mind tissues from the non-cerebrum tissues [6].

It plans to consequently identify two seed regions of the brain and non-cerebrum by utilizing a cover created by morphological operations. At that point, the seed locales were extended utilizing 2D district developing calculation, in light of the general cerebrum life structures data. Skull stripping MR cerebrum pictures utilizing anisotropic dissemination separating and morphological preparing is depicted by Gao and Xie [7]. Automatic skull stripping utilizing image form and a strategy to segment the brain from MRI human head sweeps were produced in [8], which utilizes morphological operations and joined segment investigation to recognize the cerebrum in T1-weighted MR cerebrum images.

Skull-stripping brain MR images has been challenging due to the complexity of human brain structure in both health and disease across a large number of subjects. Nevertheless, many researchers have proposed different methods worldwide. One famous approach is the brain extraction tool (BET) developed by Smith [9] employs a deformable model that advances to fit the brains surface by the utilization of an arrangement of locally versatile model powers. BET makes a force based estimation of the mind and non-cerebrum edge, decides the focal point of gravity of the head, characterizes a starting circle taking into account the focal point of gravity, and extends the circle until it achieves the mind edge. It has two flexible parameters, fragmentary force edge and limit angle. BET produces the brain volume smoother than alternate systems and regularly incorporates extra noncerebrum tissues. This calculation was tried with T1 and T2weighted images. In any case, BET has failed to separate the brain region in the bottom axial slices thus failed to extract the brain regions. ROBEX [10] method combines the discriminative and generative model to achieve the final results. The discriminative model is a random forest classifier, trained to detect the brain boundary and the generative model is a point distribution model that ensures that the result is plausible. When a new image is presented to the system to find the contour with highest likelihood in accordance with the discriminative model. There are software packages which are used for brain segmentation. These packages usually contain a set of skull stripping, intensity non uniformity correction and segmentation methods. One famous approach is the brain surface extractor (BSE) [11], which is developed based on the combination of edge detectors and morphological operators in their approach, a Marr Hildreth edge detector is first used to identify anatomical boundaries, followed by a sequence of morphological operators to separate connected tissues into individual component regions.

In this regard, the paper presents a new skull stripping algorithm .The flow of this paper is as follows. Section II discusses about the proposed methodology. Section III computation of the threshold value is based on the knowledge of intensity distribution of brain and non-brain tissues in brain MR images. In Section IV presents the using of IRF. In section $\mathrm{V}$ discuss about fuzzy morphology operations, opening and closing which are used to increase the robustness of the method. Section VI discuss the performance of the proposed method, along with the related existing skull stripping methods, BET, BSE, and ROBEX, is extensively studied on a set of both synthetic and real brain MR images qualitatively and quantitatively using standard segmentation validity metrics, and finding area fraction of brain.

\section{Proposed Methodology}

The proposed skull stripping algorithm consists of series of continuous steps enumerated below:

- Nonsensical Filter

- Binarization by Ots's threshold.

- Largest Connected Component Selection

- Fuzzy Morphological Operators.

- Region-Based Binary Mask Extraction

\subsection{Nonsensical Filter}

In MRI Brain images, vicinity of commotion, sporadic shapes, and low contrast lead to have a troublesome errand in brain tumor detection. In the pre-preparing stage, the greater part of these undesirable properties is wiped out utilizing suitable filters. In the skull-stripping strategy, the precise recognition of the shapes is urgent. In this way, it is critical to utilize a filter that can catch up the non-cerebrum tissues in a precise way. Likewise, it is just as vital to gauge the dark level dissemination unequivocally. In this admiration, in this paper, we propose a nonsensical filter in view of the Gregory-Leibniz boundless arrangement that ascertains the $\pi$ esteem with high exactness [12]

$\pi=4 \sum_{k=0}^{\infty} \frac{(-1)^{k}}{2 k+1}=\frac{4}{1}-\frac{4}{3}+\frac{4}{5}-\frac{4}{7}+\frac{4}{9}-\frac{4}{11} \ldots$

convolution operation is utilized amid filtration. The yield picture $\mathrm{g}(\mathrm{x}, \mathrm{y})$ is the aftereffect of the convolution operation between the original image $f(x, y)$ with $m$ columns and $n$ lines and the kernel $\pi(\mathrm{x}, \mathrm{y})$ with $2 \mathrm{w}+1$ columns, $2 \mathrm{~h}+1$ lines.At the same time, the $\pi$ veil isolates the picture intensities so it enhances the edge location abilities of the proposed method. Figure 1a shows an original image and fig 1.c is aresult of filteration . 


\section{International Journal of Science and Research (IJSR) \\ ISSN (Online): 2319-7064}

Index Copernicus Value (2013): 6.14 $\mid$ Impact Factor (2014): 5.611

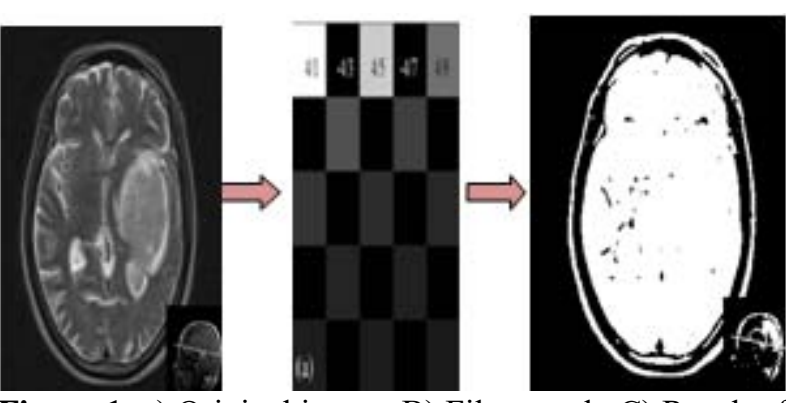

Figure 1: a) Original image, B) Filter mask, C) Result of filtration

\subsection{Binarization via Otsu's Threshold}

In binarization, the grey-level image is converted into a binary image. Here, the gray level estimation of every pixel in the filtration image at fig 1.c is ascertained, and, if the value is above the global threshold, then the pixel value is set to a binary value one; or else, it is set to zero .To separate cerebrum MR picture into two classes, one class is foundation: shaped by the low power pixels comparing to air, some portion of cerebro spinal liquid; and alternate class is made out of forefront tissues including the gray matter (GM) and White matter (WM) of the mind as appeared in the Fig 2.The fundamental issue in picture .Among various method based on threshold, there are method in view of worldwide edge and neighborhood edge. Otsu's method [13] is a global binarization method also, utilize the image variance

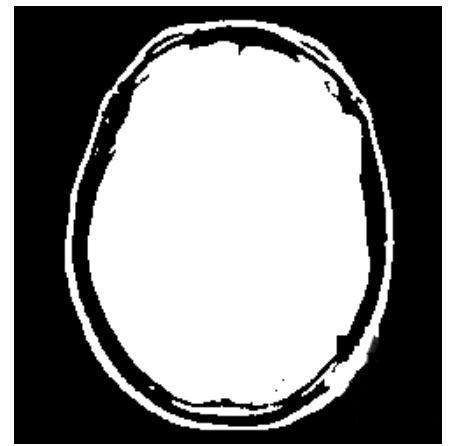

Figure 2: Binary image

\subsection{Largest Connected Component Selection}

By taking the assumption that brain is the largest connected structure inside the head we select the component whose area is the biggest. Figure 3, shows the result of this operation.

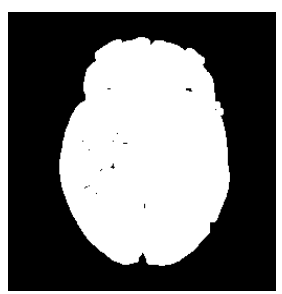

Figure 3: largest connected components

\subsection{Morphological Operation}

The morphological operators are then connected on the binarized image. The fundamental capacity of the morphological operators is to expel commotion from the image. The morphological operators, for example, opening, erosion, closing and dilation are utilized as a part of the proposed procedure. Later on in the mid-1970s and mid1980 s, it was summed up to greyscale image that require more complex numerical operations. All the while the operators are stretched out to new operators. Subsequently, scientific morphology increased much acknowledgment and is utilized generally as a part of the image handling application. Numerical morphology is depicted totally by set operation, for example, union, crossing point, distinction and supplement. Set is a gathering of pixels in a picture. binary morphology depends just on set membership and does not take into account the grey value or colour of the image pixel. Morphological operators transform the original image into another image of certain shape and size, also known as structuring element. Scientific morphology gives a way to deal with break down the geometric qualities of pictures and has been generally utilized as a part of picture edge identification, division, commotion concealment. Fluffy scientific morphology extends the twofold morphological operators to gray level images. In binary morphology, fuzzy erosion, dilation, opening and closing are present. In a similar way in fuzzy morphological operations [14], union operation is replaced by a maximum operation and intersection operation is replaced by a minimum operation. Similarly in fuzzy morphological operations, union operation is supplanted by a most extreme operation and intersection operation is supplanted by a base operation. Morphological operators are utilized to locate the morphological slope or to denoise the image. The impact of disintegration and expansion operations is better to find the Image edge by taking the distinction between the enlarged picture and dissolved picture. Instead of disintegration and expansion, opening and shutting operations perform better in denoising the pictures. Using Fuzzy closing and opening operations are characterized utilizing fuzzy erosion and dilation using equation below:

Opening:

$\mathrm{O}(\mathrm{A}, \mathrm{B})(\mathrm{x})=\mathrm{D}(\mathrm{E}(\mathrm{A}, \mathrm{B})(\mathrm{x}), \mathrm{B}(\mathrm{x}))=\mathrm{D}(\inf \min [1,1+\mathrm{A}(\mathrm{x}) \mathrm{B}(\mathrm{x})], \mathrm{B}(\mathrm{x}))(1)$

Closing:

$\mathrm{C}(\mathrm{A}, \mathrm{B})(\mathrm{x})=\mathrm{E}(\mathrm{D}(\mathrm{A}, \mathrm{B})(\mathrm{x}), \mathrm{B}(\mathrm{x}))=\mathrm{E}(\sup \max [0, \mathrm{~A}(\mathrm{x})+\mathrm{B}(\mathrm{x}-1)], \mathrm{B}(\mathrm{x}))(2)$

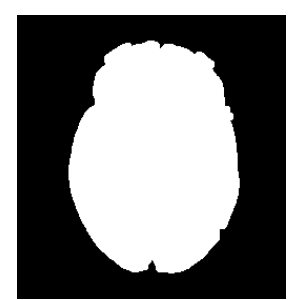

Figure 4: Output of fuzzy morphological

\subsection{Brain Mask Extraction}

The brain is extracted by performing AND operation between MRI brain images, Figure1.a, with binary mask, Figure 4 is performed by considering the properties of each block that satisfy some criteria. Thus, we have obtained a brain MRI image with its brain cortex stripped 


\section{International Journal of Science and Research (IJSR) \\ ISSN (Online): 2319-7064}

Index Copernicus Value (2013): 6.14 | Impact Factor (2014): 5.611

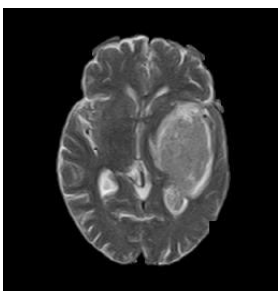

Figure 5: Skull stripped result

\section{Experimental Results and Discussion}

Data used in this thesis are T1-weighted and T2-weighted head MRI scans and experiments have been done on the axial views of the MRI scans. We validated the proposed framework using three different datasets:

- Real tumorous brain MRI scans from Mansoura Hospital.

- The medical images acquired from Mansoura Hospital were stored in a DICOM (Digital Imaging and Communications in Medicine) format which is the standard image format in the medical field.

- Real normal (healthy) MR images from Internet Brain Segmentation Repository (IBSR) [15].

Based on these outcomes the metrics are defined as follows, Jaccard similarity index for two sets is defined as the size of intersection of the two sets divided by the size of their union as given in equation (3).

$$
J S I=\frac{\left|G_{Y} \cap O_{M}\right|}{\left|G_{T} \cup O_{M P}\right|}=\frac{T_{P}}{\left(T_{P}+F_{P}+F_{N}\right)} .
$$

A Jaccard similarity index value of indicates a perfect agreement between the two sets and indicates no agreement between sets. The sensitivity measures the fraction of true positives that are correctly detected and it is defined as in equation (4).

$$
\text { Sensitivity }=\frac{T_{P}}{\left(T_{P}+F_{N}\right)} \ldots \ldots \ldots \ldots
$$

The specificity measures the fraction of true negatives that are correctly detected. Specificity is defined as in equation (5).

$$
\text { Specificity }=\frac{T_{N}}{\left(T_{N}+F_{P}\right)} \ldots \ldots \ldots .
$$

By evaluating the results of the above four validation metrics, we can conclude how the proposed method is efficient in skull stripping, tumor detection and segmentation in brain MR images when compared against ground truth. The skull stripping method is tested on IBSR and Mansoura university datasets. The qualitative and quantitative visual results of skull stripping method are presented as follows. Table 1shows the result of the proposed skull stripping method on IBSR datasets only because we have not golden standard of our real images. Figure 6 show the result of skull stripping by proposed algorithm on IBSR data sets. We calculated the Dice similarity score, Jaccard similarity index, specificity and sensitivity of the skull stripping result using manually obtained ground truth .A score of represents perfect overlap while represents no overlap. Sensitivity measures how well the performance of skull stripping method is in avoiding removal of brain tissues together with non-brain tissues. On the other hand, specificity measures how well the performance of the method on not wrongly classifying nonbrain tissues as brain tissues. Larger value of sensitivity indicates the more accurate the skull stripping method is. But, if in case the skull stripping technique includes nonbrain tissues in the final result rather than avoiding them sensitivity remains high. So, sensitivity has to be coupled with specificity to measure the accuracy of the skull stripping method. Generally, an accurate and robust skull stripping technique must have good performance for all multiple performance metrics.

Skull stripping algorithm developed in this study is compared to the well-known algorithms Brain Surface Extractor (BSE) [11], Brain Extraction Tool (BET) [9], according to Jaccard similarity index, sensitivity and specificity. Because the other studies give their results in Jaccard index, performance comparison results are given as Jaccard index. All methods are evaluated based on their performance on IBSR database [15] that contains both T1-weighted MR images and their manual segmentations. In Table I, performance of the developed algorithm is compared to the other algorithms. The results are available in all referenced studies, of the IBSR database. From the scores in Table I, it can be seen that that our algorithm performs better the BSE whereas it outperforms other methods.

Table 1: Comparison of the skull stripping method

\begin{tabular}{|c|c|c|c|}
\hline & BET & BSE & Developed algorithm \\
\hline Jaccard & 0.71 & 0.93 & 0.98 \\
\hline Sensitivity & 0.99 & 0.83 & 0.99 \\
\hline Specitivity & 0.99 & 0.99 & 0.99 \\
\hline
\end{tabular}
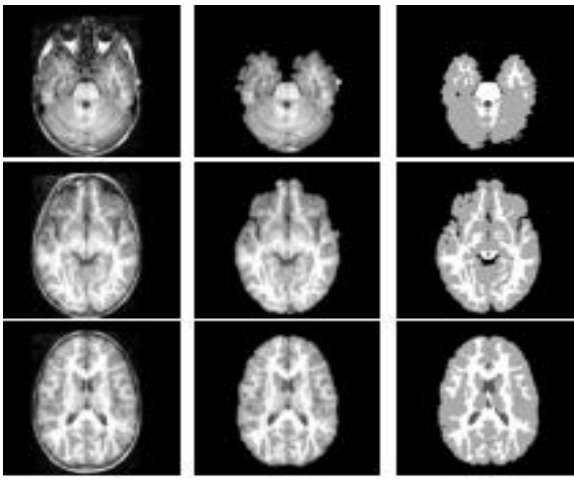

Figure 6: Skull stripping IBSR brain images. Columns from left to right a) original image .b) skull stripping by proposed algorithm, c) ground truth provided by IBSR

\section{Conclusions}

This paper proposes a fuzzy morphological algorithm and irrational filter to implement skull stripping technique. Promising results demonstrate that proposed algorithm show a good performance and can complete the segmentation task successfully with simple step and little time .and is of strong robustness as well as high accuracy rate. The proposed method does not require any initial user intervention or parameters and thus qualify to be an automatic method. Exiting method such as BET and BSE also require parameter adjustment. BET and BSE and ROBEX fail to segment brain 


\section{International Journal of Science and Research (IJSR) \\ ISSN (Online): 2319-7064 \\ Index Copernicus Value (2013): 6.14 | Impact Factor (2014): 5.611}

and non brain image .Finally Results ensure that the proposed method is suitable on both real image and also for IBSR image.

\section{References}

[1] Sajjad Mohsin, Sadaf Sajjad, Zeeshan Malik, and Abdul Hanan Abdullah, "Efficient way of skull stripping in MRI to detect brain tumor by applying morphological Operations, after detection of false background" , IJIET Vol.2, No.4, August 2012

[2] Rosniza Roslan, Nursuriati Jamil and Rozi Mahmud, "Skull Stripping Magnetic Resonance Images Brain Images: Region growing versus Mathematical Morphology" , International Journal of Computer

[3] S.A.Hojjatoleslami and J.Kittler, "Region Growing: new approach", IEEE Trans. Image Processing, Vol7, pp1079-1084, 1998

[4] . F.Segonne, A.M.Dale, E. Busea, M.Glessner, D. Salat,H.K Hann and B.Fischl, "A hybrid approach to the skullstripping problem in MRI" , Neuro Image 22 (2004), pp1060-1075

[5] Brummer ME, Mersereau RM, Eisner RL, Lewine RRJ, CaesllesV, Kimmel R, Sapiro G: Automatic detection of brain contours inMRI datasets. IEEE Trans Image Process 12(2):153-166, 1993

[6] Zhuang AH, Valentino DJ, Toga AW: Skull stripping magnetic resonance images using a model-based level sets. NeuroImage 32(1):79-92, 2006

[7] Gao J, Xie M: Skull stripping MR brain images using anisotropic diffusion filtering and morphological processing, Proc.International Symposium on Computer Network and MultimediaTechnology, Wuhan 1:1-4,2009

[8] Somasundaram K, Kalavathi P: A hybrid method for automatiskull stripping of magnetic resonance images (MRI) of human

[9] head scans. Proc. International Conference on ComputinCommunication and Networking Technologies (ICCCNT), Karur, Tamilnadu, 1-5, 2010

[10] Smith SM: Fast robust automated brain extraction. Hum Brain Mapp 17(3):143, 2002

[11] Shattuck DW, Sandor-Leahy SR, Schaper KA, Rottenberg DA, Leahy RM: Magnetic resonance image tissue classification using a partial volume model. NeuroImage 13(5):856-876, 2001

[12] Shattuck DW, Sandor-Leahy SR, Schaper KA, Rottenberg DA, Leahy RM: Magnetic resonance image tissue classification using a partial volume model. NeuroImage 13(5):856-876, 2001

[13] DW, Sandor-Leahy SR, Schaper KA, Rottenberg DA, Leahy RM: Magnetic resonance image tissue classification using a partial volume model. NeuroImage 13(5):856-876, 2001

[14] Otsu N: A threshold selection method from gray level histograms. IEEE Trans Syst Man Cybern 9:6266, 1979

[15] Leahy Sinha, D. and Dougherty, E.R., Fuzzification of set inclusion: Theory and application ,Fuzzy Sets and Systems, 55, 15-42, 1993

[16] Center for Morphometric Analysis, Massachusetts General Hospital, the Internet Brain Segmentation Repository http://www.cma.mgh.harvard.edu/ibsr/, accessed on: November 10, 2011 